\title{
Pleural Effusion in Limited Cutaneous Systemic Sclerosis : A Report
}

\author{
Shivjeet Yadav ${ }^{1}$, Kuber Sharma ${ }^{2}$, Vikram Kumar ${ }^{2}$, Sonu Kumar Singla ${ }^{2}$, Navdeep Malik ${ }^{2}$ \\ ${ }^{1}$ Asisstant Professor, ${ }^{2}$ Resident, Department of Medicine, SGT Medical College, Gurugram
}

\begin{abstract}
Systemic sclerosis (SSc) is a chronic autoimmune multisystem disorder characterized by endothelial dysfunction and fibroblast dysfunction, which results in progressive fibrosis of the skin and internal organs more frequently the lungs and gastro intestinal tract. Pulmonary involvement is common in the course of SSc, with Interstitial Lung Disease (ILD) and Pulmonary Arterial Hypertension (PAH) along with pleural effusion being the leading causes of death. Here we report, case of an elderly male patient presenting with cutaneous SSc with multiple uncommon pulmonary manifestations like ILD with right sided pleural effusion with hepatic cyst.
\end{abstract}

Keywords: Interstitial lung disease, Pulmonary arterial hypertension, Scleroderma, Usual interstitial pneumonia

\section{Report}

A 55-year-old male presented to the Department of Medicine, SGT Medical College and Hospital, with complaints of generalized swelling with malygia since 2 months, stiffness and darkening of skin with rash since 15 days, cough with expectoration and progressive breathlessness since three months, difficulty in swallowing, and loss of appetite . On examination- Scleroderma involving both the upper limbs, face, trunk and lower limbs was present. Sclerodactyly of all fingers was present with salt and pepper appearance of skin over the scalp, fore head, upper back, chest, trunk and limbs(figure) and bilateral basal crackles were present. Abdomen was

\section{Corresponding Author:}

\section{Dr Sonu Kumar Singla}

Resident, Department of Medicine, SGT Medical

College, Gurugram

S.k.singla24@gmail.com

98760333265 soft and non-tender with hepatomegaly as well as splenomegaly. Routine lab investigations revealed$\mathrm{Hb}$ of $9.1 \mathrm{~g} \%$, total count- 10,000 cells/ cumm, platelets- 2.4 lac cells/ cumm, RBS- $147 \mathrm{mg} / \mathrm{dl}$, LFT's, RFT's and serum electrolytes within normal limits. Chest X-Ray showed right reticulo-nodular opacities with basal predominance. Spirometry showed restrictive defect with an FEV1/ FVC of $88, \mathrm{FEV} 1=70 \%$ and $\mathrm{FVC}=60 \%$. Two dimensional ECHO revealed normal chambers with LVEF 55\%. CECT showed mild hepatomegaly with hepatic cyst with right pleural effusion with small retroperitoneal lymph nodes. Connective tissue profile was sent. ANA and Anti Scl-70 were positive, whereas RA factor, anti- RNP, anti-Sm, c-ANCA and p-ANCA were negative. Skin biopsy (right arm lesion)- Dermis showed homogenous appearing thickened collagen bundles, consistent with scleroderma . 


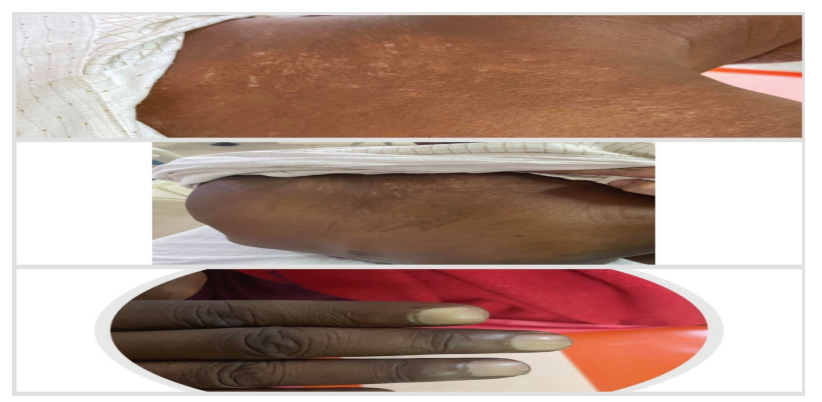

Figure:sclerodermal changes over limbs.

\section{Discussion}

Systemic Sclerosis (SSc) is a connective tissue disorder of unknown aetiology affecting arterioles, micro vessels and fibroblasts resulting in vascular obliteration, collagen accumulation, scarring (fibrosis) and abnormalities of immune system ${ }^{[1]}$. SSC is more commonly seen in middle aged females between 45-55 years of age. It's prevalence in Asia is between 20-50 million ${ }^{[2]}$. Lung involvement is common in course of the disease, with ILD and pleural effusion being most frequent. The other pulmonary manifestations are aspiration pneumonia, pleural effusion, spontaneous pneumothorax and malignancy [2]. SSc patients with ILD and pleural effusion have a 5 fold increase in mortality rate. The prevalence of SSc-ILD with PAH is $18-22 \%{ }^{[3]}$. SSc-ILD usually presents with Nonspecific interstitial pneumonia (NSIP) pattern, but diffuse SSc showing UIP pattern is uncommon with an incidence of only $11 \%$. The occurrence of pleural effusions is also rare and has been reported in only $7 \%$ of the cases ${ }^{[4]}$.

Depending on the degree and extent of skin involvement SSc can be classified as:

Limited cutaneous (Lc SSc): Skin thickening restricted to sites distal to the elbows and knees, but may involve face and neck.

CREST syndrome: Subset of limited cutaneous SSc with prominent calcinosis, Raynaud's phenomenon, esophageal dysmotility, sclerodactyly and telangiectasia.
Diffuse cutaneous (Dc SSc): Skin thickening over the trunk and proximal extremities in addition to distal extremities and face.

Overlap SSc: Skin changes and other characteristic features of SSc coexisting with features of another connective tissue disease, such as systemic lupus erythematosus, rheumatoid arthritis, dermatomyositis or Sjogren's syndrome.

SSc sine scleroderma: Characteristic internal organ complications like pulmonary fibrosis or renal, cardiac or gastrointestinal disease, but no apparent skin involvement ${ }^{[5]}$

Criteria for classification of Systemic Sclerosis:

\section{Systemic Sclerosis (Scleroderma)} Classification Criteria: These criteria were established in 1980 by the American Rheumatism Association (now known as the American College of Rheumatology $)^{[6]}$.

Requirements: Either the sole major criterion or two or more of the minor criteria.

Major Criterion: Proximal scleroderma: Symmetrical thickening, tightening and induration of the skin of the fingers and the skin proximal to the metacarpophalangeal or metatarsophalangeal joints. These changes can involve the entire limb, face, neck and trunk.

Minor Criterion: Sclerodactyly: Induration and tightening of the skin of the fingers.

Digital ischemia: Manifested by digital pitting scars or atrophy of finger pads.

Bibasilar pulmonary fibrosis: Reticular or reticulonodular densities most pronounced in the basilar areas of the lungs on chest X-ray (CXR). This may produce the appearance of "honeycomb lung" and must not be due to a primary pulmonary disease. 
But the 1980 ACR criteria were not sensitive enough to identify patients with early disease or limited cutaneous SSc. To address this issue, a newer classification criterion was developed in 2013 by the joint ACR-EULAR committee [Table/Fig-6]. Based on the newer criterion- Skin thickening of the fingers extending proximal to the metacarpophalangeal joints is sufficient for a patient to be classified as having scleroderma. If this is not present, seven other additive items are considered, with varying weights or scores for each. Patients with a total score of $\geq 9$ are classified as having definite scleroderma ${ }^{[7]}$

Investigations in systemic sclerosis are pulmonary function test which shows restrictive pattern and high resolution computerised tomography (HRCT) - NSIP pattern is most common. However, a UIP pattern showing peripheral and basilar predominant reticulation and honeycombing without significant ground glass opacities, can also be seen, but is more common in patients with $\mathrm{LcSSc}$ [8]. Immunosuppressants are the main stay of treatment for SSc. Cyclophosphamide, Mycophenolate Mofetil, Corticosteroids (usually in combination with the above drugs) are the preferred drugs. Other therapies under investigation are Bosentan (Endothelin receptor antagonist), Imatanib (tyrosine kinase inhibitor), Rituximab (a monoclonal antibody directed against the CD20 antigen on the surface of B lymphocytes) and Stem cell transplant [9]. Lung transplantation is considered in very rare cases.

\section{Conclusion}

We have thus presented a case of cutaneous systemic sclerosis (cSSc) with multiple uncommon pulmonary manifestations like right sided pleural effusion. The diagnosis of systemic sclerosis may at times be difficult, due to the overlapping features with other connective tissue diseases. There is also increased risk of organ damage early in the course of the disease even when the patient is asymptomatic.
Hence, timely diagnosis and management is of utmost importance.

Ethical Clearance- taken from institutional committee

\section{Source of Funding- Self}

\section{Conflict of Interest - Nil}

\section{References}

[1] Mouthon L, et al. Interstitial lung disease in systemic sclerosis. Rev Mal Respir. 2009;26:e1e11. [Google Scholar]

[2] Mouthon L, Allanore Y, Cabane J, Hachulla E. Maladies systemiques. Paris: Flammarion Medecine-Sciences; 2006. Scleroderdermie systemique. In: Guillevin L, Meyer O, Piette J, Sibilia J, editors. [Google Scholar]

[3] Chang B, Wigley FM, White B, et al. Scleroderma patients with combined pulmonary hypertension and interstitial lung disease. J Rheumatol. 2003;30:2398-405. [PubMed] [Google Scholar]

[4] Thompson AE, Pope JE. A study of the frequency of pericardial and pleural effusions in scleroderma. Br J Rheumatol. 1998;37:1320-23. [PubMed] [Google Scholar]

[5] Gabrielli A, Avvedimento EV, Krieg T. Scleroderma. N Engl J Med. 2009;360(19):19892003. [PubMed] [Google Scholar]

[6] Preliminary criteria for the classification of SSc. Arthritis Rheum. 1980;23(5):581-90. [PubMed] [Google Scholar]

[7] Van den Hoogen F, et al. 2013 Classification criteria for systemic sclerosis. Arthritis Rheum. 2013;65:2737-47. [PMC free article] [PubMed] [Google Scholar]

[8] Goldin JG, Lynch DA, Strollo DC, et al. Highresolution CT scan findings in patients with symptomatic scleroderma-related interstitial lung 
disease. Chest. 2008;134:358-67. [PubMed] [Google Scholar]

[9] Scholand, et al. Interstitial lung disease in systemic sclerosis: diagnosis and management. Rheumatology. 2012;S1:008. [Google Scholar] 